\title{
Microvascular destruction identifies murine allografts that cannot be rescued from airway fibrosis
}

\author{
Ashok N. Babu, ${ }^{1}$ Tomohiro Murakawa, ${ }^{2}$ Joshua M. Thurman, ${ }^{3}$ Edmund J. Miller, ${ }^{4}$ Peter M. Henson, ${ }^{5}$ \\ Martin R. Zamora, ${ }^{6}$ Norbert F. Voelkel, ${ }^{6}$ and Mark R. Nicolls ${ }^{7}$
}

\begin{abstract}
${ }^{1}$ Department of Surgery, University of Colorado at Denver and Health Sciences Center, Denver, Colorado, USA. ${ }^{2}$ Department of Cardiothoracic Surgery, University of Tokyo, Tokyo, Japan. ${ }^{3}$ Division of Renal Diseases and Hypertension, University of Colorado at Denver and Health Sciences Center, Denver, Colorado, USA. ${ }^{4}$ The Feinstein Institute of Medical Research, Northshore-Long Island Jewish Medical Center, Manhasset, New York, USA. ${ }^{5}$ Division of Pulmonary Medicine, National Jewish Medical Center, Denver, Colorado, USA. ${ }^{6}$ Divison of Pulmonary Sciences and Critical Care Medicine, University of Colorado at Denver and Health Sciences Center, Denver, Colorado, USA. ${ }^{7 D i v i s i o n}$ of Pulmonary and Critical Care Medicine,
\end{abstract} VA Palo Alto Health Care System, Palo Alto, California, USA

\begin{abstract}
Small airway fibrosis (bronchiolitis obliterans syndrome) is the primary obstacle to long-term survival following lung transplantation. Here, we show the importance of functional microvasculature in the prevention of epithelial loss and fibrosis due to rejection and for the first time, relate allograft microvascular injury and loss of tissue perfusion to immunotherapy-resistant rejection. To explore the role of alloimmune rejection and airway ischemia in the development of fibroproliferation, we used a murine orthotopic tracheal transplant model. We determined that transplants were reperfused by connection of recipient vessels to donor vessels at the surgical anastomosis site. Microcirculation through the newly formed vascular anastomoses appeared partially dependent on VEGFR2 and CXCR2 pathways. In the absence of immunosuppression, the microvasculature in rejecting allografts exhibited vascular complement deposition, diminished endothelial CD31 expression, and absent perfusion prior to the onset of fibroproliferation. Rejecting grafts with extensive endothelial cell injury were refractory to immunotherapy. After early microvascular loss, neovascularization was eventually observed in the membranous trachea, indicating a reestablishment of graft perfusion in established fibrosis. One implication of this study is that bronchial artery revascularization at the time of lung transplantation may decrease the risk of subsequent airway fibrosis.
\end{abstract}

\section{Introduction}

The major obstacle to survival following lung transplantation is the bronchiolitis obliterans syndrome (BOS) (1), which is clinically defined by a decline in ventilatory function and histologically characterized by fibro-obliteration of small airways (2). Despite identification of risk factors for the development of BOS such as rejection and $\mathrm{CMV}$ infection, the etiology of the fibroproliferative changes associated with BOS remains unknown (3). Recent autopsy studies from Luckraz and colleagues demonstrate a marked loss of microvasculature in nonoccluded small airways from BOS lungs, suggesting airway ischemia as a preceding condition to airway fibrosis $(4,5)$. Accordingly, some have hypothesized that chronic airway ischemia and hypoxia could contribute to airway fibro-obliteration following lung transplantation (6-8).

To explore the role of alloimmune rejection and airway ischemia in the development of fibroproliferation, we used a murine orthotopic tracheal transplant (OTT) model. Allogeneic OTTs have previously been used as a model of lymphocytic bronchitis, the large airway correlate of BOS, developing both epithelial metaplasia and subepithelial fibrosis (9). In the present study, we delineate the interactions

Nonstandard abbreviations used: $\mathrm{B} 6, \mathrm{C} 57 \mathrm{BL} / 6$; BOS, bronchiolitis obliterans syndrome; CD40L, CD40 ligand; CXCR2, CXC chemokine receptor 2; OTT, orthotopic tracheal transplant; $\mathrm{pO}_{2}$, partial pressure of oxygen.

Conflict of interest: Joshua M. Thurman is the recipient of an Amgen Junior Faculty Research Award (research funding of $\$ 60,000$ per year).

Citation for this article: J. Clin. Invest. 117:3774-3785 (2007). doi:10.1172/JCI32311. of host and recipient blood vessels after OTT and demonstrate that the resultant time-dependent loss of vascular perfusion in allografts corresponds to the presence of tissue hypoxia. Critical time points of rejection were then studied in a clinically relevant model to demonstrate the importance of functional microvasculature in the prevention of epithelial loss and fibrosis due to rejection.

\section{Results}

Acute rejection of orthotopic tracheal allografts leads to loss of donor-type epithelium, loss of submucosal vessels, and fibrosis by day 28 . We first sought to determine histologic differences between untreated allografts and syngeneic grafts (syngrafts; Figure 1). Syngrafts have normal architecture on day 6 following transplantation. Allografts at the same time point demonstrate inflammatory cell infiltrates of the submucosa and epithelium composed of $\mathrm{CD}^{+}$and $\mathrm{CD} 8^{+} \mathrm{T}$ cells, neutrophils, and macrophages (our unpublished observations). Twenty-eight days following transplantation, untreated allografts developed subepithelial fibrosis and loss of columnar epithelium. To explore changes in epithelial morphology and donor-recipient interactions, we used coronally sectioned tracheal specimens that included both the allograft and the recipient tracheal ends en bloc. Allograft epithelium at day 8 in the graft was still donor derived as demonstrated by $\mathrm{H}-2 \mathrm{~K}^{\mathrm{d}}$ staining. Day 12 allograft coronal sections illustrated epithelial replacement by flattened cells following graft-specific sloughing of columnar epithelium at day 10 (data not shown). There was a loss of endothelial cell staining in allografts between day 10 and day 12 . Simultaneous loss of CD $31^{+}$ 
A Day 6 syn (H\&E)

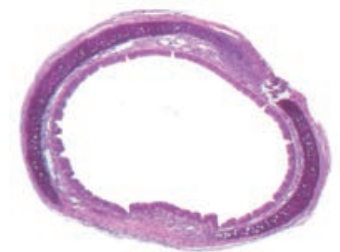

E Day 8 allo (Class I H-2K ${ }^{\mathrm{d}}$ )

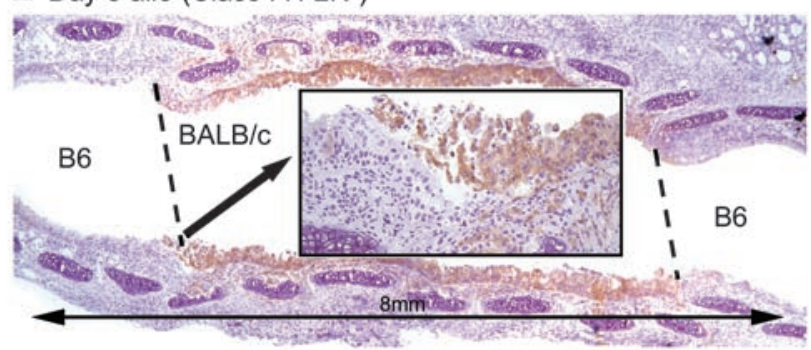

C Day 28 syn (trichrome)

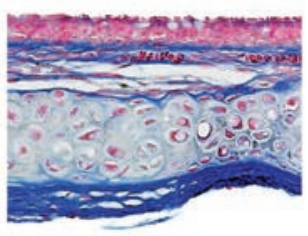

D Day 28 allo (trichrome)

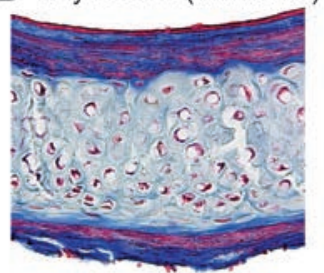

F Day 12 allo (Class I H-2K $K^{d}$ )
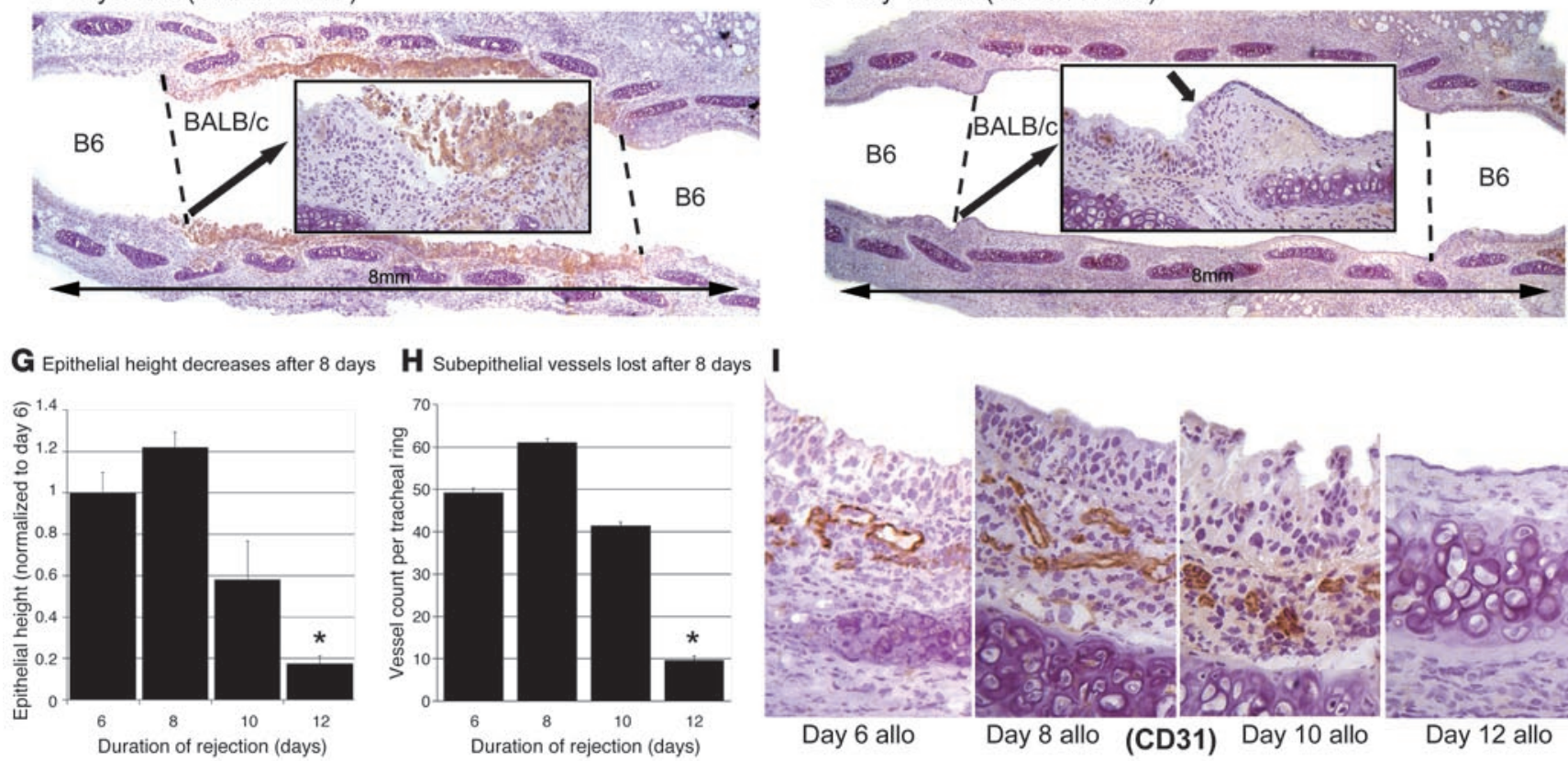

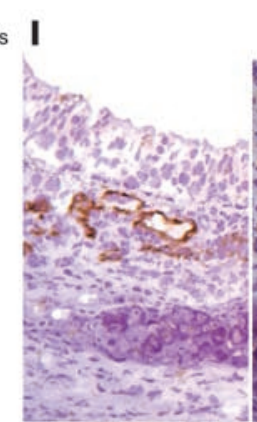

Day 6 allo

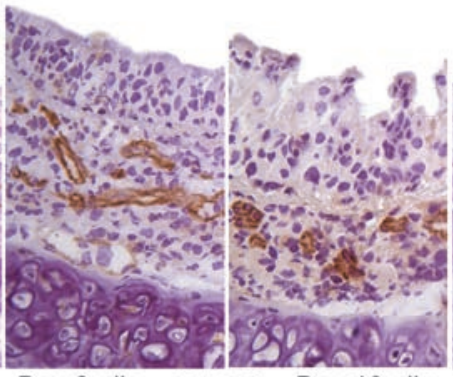

Day 8 allo (CD31) Day 10 allo

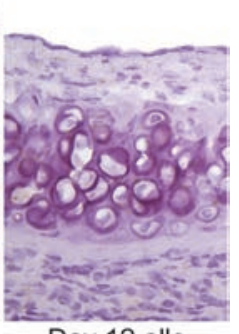

Day 12 allo

Figure 1

Loss of epithelium and subepithelial vessels after 8 days of acute rejection. (A) Radial section of syngeneic (B6 $\rightarrow$ B6) tracheal graft 6 days after transplant demonstrating normal columnar epithelium. (B) Allogeneic (BALB/c $\rightarrow B 6)$ graft with massive inflammatory cell infiltration of the subepithelium and epithelium 6 days following transplant. (C and D) Masson's trichrome stain demonstrates subepithelial fibrosis and epithelial changes at 28 days in allografts as compared with normal histology in syngeneic grafts. (E) Coronal section of tracheal allograft at 8 days $(n=4)$ stained for $\mathrm{MHC}$ class I H-2Kd $(B A L B / c)$ demonstrates donor-type columnar epithelium in the graft without staining of recipient epithelium. (F) Coronal section of allograft at 12 days immunostained for MHC class I H-2K demonstrates replacement of columnar epithelium with flattened epithelium (short arrow). (G) Morphometric analysis of epithelial height demonstrates epithelial height loss between 8 days and 12 days. (H and I) Radial sections of tracheal allografts and corresponding vessel counts demonstrate loss of subepithelial vessels after 8 days of rejection. ${ }^{*} P<0.01$ versus all other groups; $n=4-5$ for all groups. allo, allogeneic; syn, syngeneic. Original magnification, $\times 4$ (A, B, H, and I); $\times 20$ (C and D).

structures and of epithelia (an event tightly linked to fibrosis) suggested that the 2 events were related and further study of perfusion status of the vessels was therefore warranted.

Tracheal microvasculature is bighly organized based on cartilaginous anatomy. The trachea consists of cartilage C-rings, which are separated axially as well as in the opening of the $\mathrm{C}$ by membranous trachea. Tissue histology was evaluated after ventral division and then flattening of the tracheal tube under a coverslip as a whole mount. Figure 2 shows angiograms of the same naive trachea by 2 different techniques, FITC-tagged lectin vascular perfusion in vivo and tracheal whole-mount CD31 immunostaining ex vivo. Though CD31 can also be expressed by lymphatic endothelium, use of the lymphatic specific marker LYVE-1 demonstrated that the majority of $\mathrm{CD} 31^{+}$structures in this model were not lymphatics (our unpublished observations).

Allogeneic and syngeneic grafts were perfused by day 6 following transplantation. To determine how and when these grafts were perfused with blood, allografts and syngrafts were harvested on days 2, 4, and 6 following implantation (Figure 3). Prior to sacrifice, animals received intravenously administered FITC-conjugated tomato lectin to bind and identify perfused blood vessels. Excised tracheae were also stained as whole tissues for CD31 to identify blood vessels regardless of their perfusion status. At day 2, both syngrafts and allografts had a complete lack of perfusion, though there was a vascular network identified by CD31 staining. Perfusion in both allografts and syngrafts was first evident on day 4 and was complete by day 6 . This relatively expeditious and complete perfusion of the grafts led us to query whether the donor vessel network was retained and became continuous with the recipient animal's vessels.

Allografts and syngrafts reperfuse by connections between recipient and donor blood vessels at the level of the anastomosis. To assess whether graft reperfusion occurs by neovascularization of the graft or by reperfusion of the donor vascular network via connections with recipient vessels, we used FVB strain transgenic mice expressing 


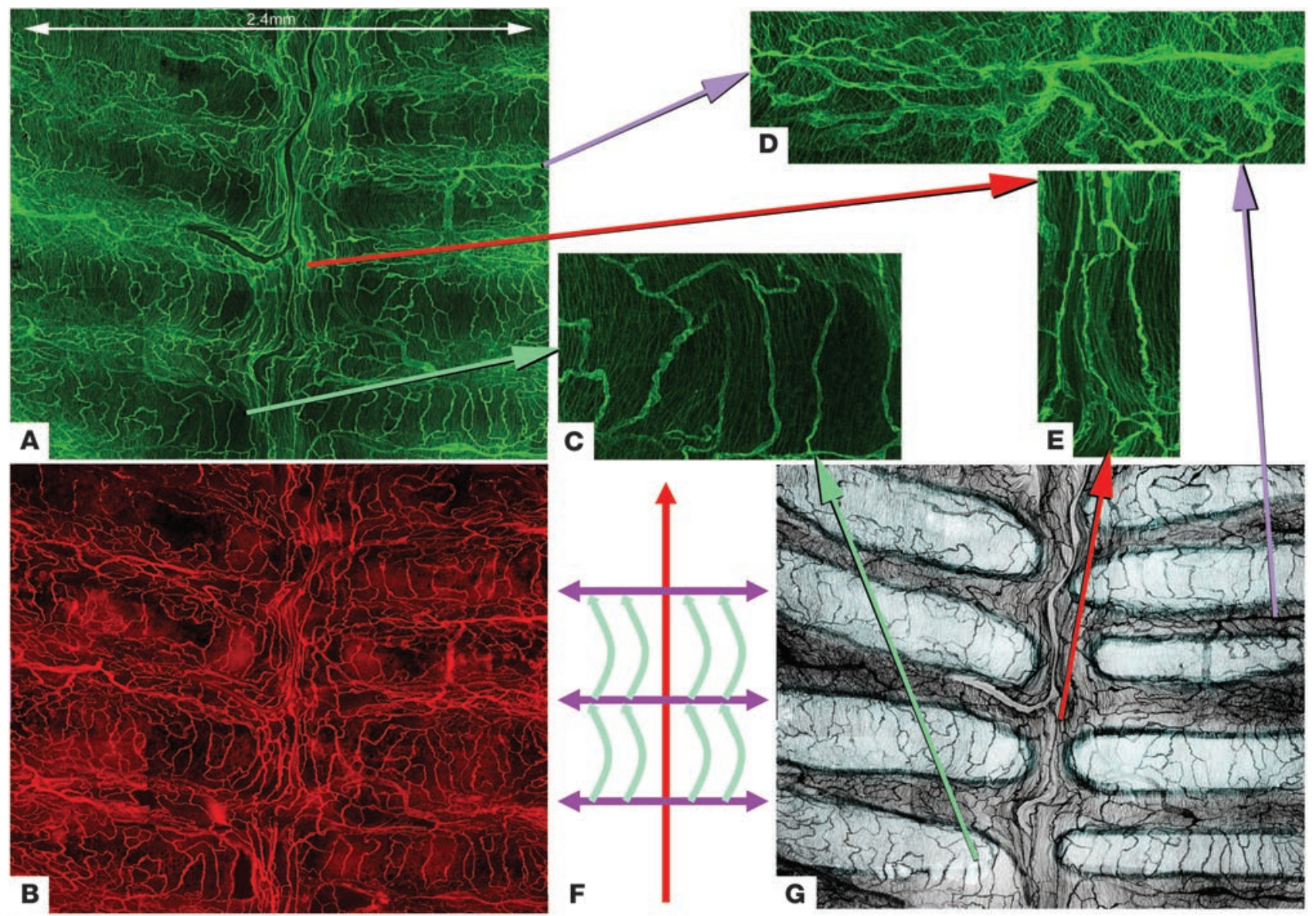

Figure 2

Tracheal microvasculature is organized based on cartilaginous anatomy. (A) Following i.v. injection of FITC-conjugated (green) tomato lectin while still alive, naive animals were sacrificed and tracheae whole mounted to visualize the tracheal vasculature. (B) These same tracheae underwent immunostaining for CD31 (endothelial cell antigen) with a Cy3 (red) secondary antibody after excision to identify all vessels regardless of perfusion status. (C) Magnified image of vessels originating from the intercartilaginous vessels (D) that span the cartilage rings. (D) Transverse vessels carrying blood away from the midline to the intercartilaginous trachea to supply cartilage-spanning vessels in C. (E) Axial direction vessels in the midline membranous trachea, which appear to be major blood highway for trachea. $(\mathbf{F})$ Schematic depiction of tracheal blood flow, which is color-coordinated with previous cutout images. (G) Cartoon version of image A demonstrating cartilage rings in light blue and intercartilaginous or membranous regions in dark gray. Original magnification, $\times 20$.

$\beta$-galactosidase on the endothelial cell-specific Tie2 promoter. Allografts $(\mathrm{BALB} / \mathrm{c} \rightarrow \mathrm{FVB}[$ Tie $2 / \beta$-galactosidase]) and syngrafts $(\mathrm{FVB}[\mathrm{WT}] \rightarrow \mathrm{FVB}[$ Tie $2 / \beta$-galactosidase $])$ were sacrificed at day 6 , and animals were perfused with red-colored India ink via the aorta. Specimens were evaluated as whole mounts (Figure 3D). In both allografts and syngrafts, graft vessels were only red in color - proving that they were perfused but not composed of recipient endothelial cells. Vessels in the recipient ends were blue in color, consistent with their recipient origin. Further magnification at the anastomosis revealed that multiple small blue (recipient) vessels connect to red (donor) vessels at the anastomosis. Uniquely in the allografts, these vessels are chimeric, composed of MHC-mismatched endothelium.

Infiltration of recipient-derived $\mathrm{CD} 31^{-} /$Tie $^{+}$cells into graft occurs at 6 day. Many cells expressing $\beta$-galactosidase (and hence Tie2) but not a component of perfused vessels were observed in the allograft at day 6 (Figure 3E). These cells appeared to be interspersed among the $\mathrm{CD} 31^{+}$vessels but did not make up part of the vessel wall or express CD31 antigen. To confirm that CD31 staining was not obscured by $\beta$-galactosidase staining, we stained serial sections with CD31 alone and found that the nonvascular Tie2-expressing cells in fact did not express CD31.

Untreated allografts lose perfusion by day 10. As shown above, CD31 staining of axial sections was diminished on day 10 and disappeared by day 12 . To further study this finding, vascular perfusion experiments were performed in 3 groups of animals: syngrafts, untreated allografts, and treated allografts (anti-LFA-1 + anti-CD40 ligand [anti-CD40L] at days 8, 10, and 12. Syngrafts were perfused at all 3 time points (Figure 4), whereas allografts had complete loss of perfusion throughout the graft at both day 10 and day 12 . Animals treated with immunosuppression were comparable to those receiving syngrafts, implicating the unchecked alloimmune response in the loss of perfusion. Use of mean fluorescent intensity and percentage area of vessels per $20 \times$ field on all specimens confirmed visual findings. There was also a loss of CD31 immunoreactivity, with only small segments of $\mathrm{CD} 31^{+}$vessels remaining in the 
A

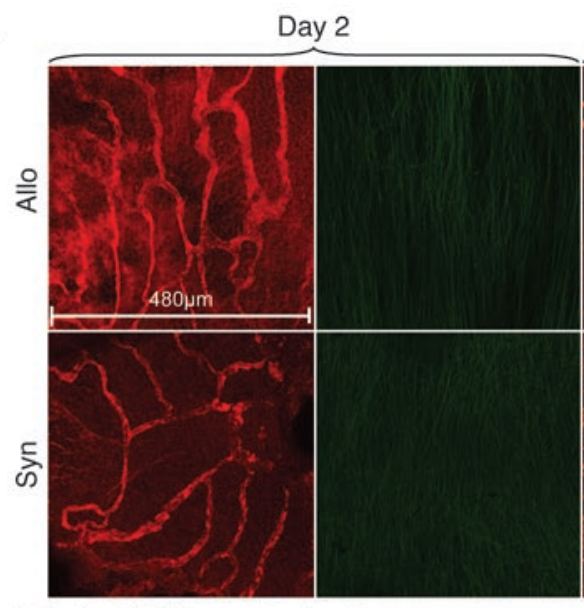

B Perfusion (MFI) Increases by d6
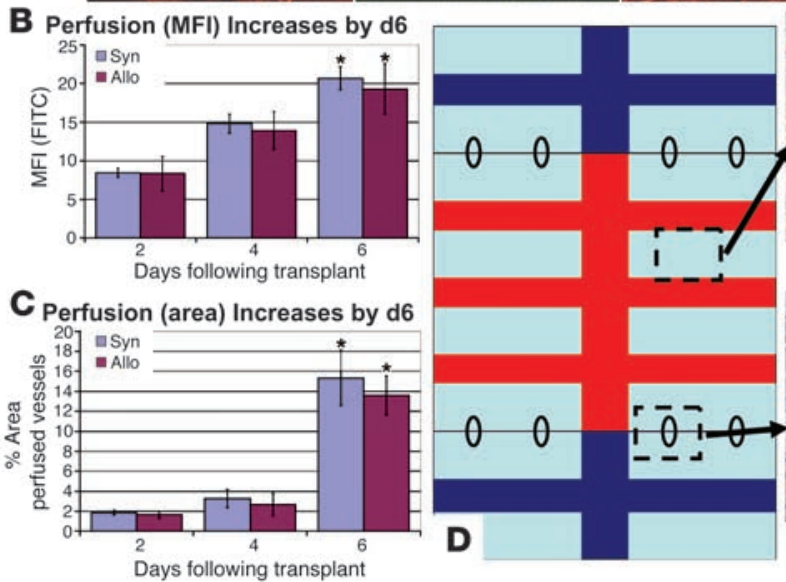

Day 4
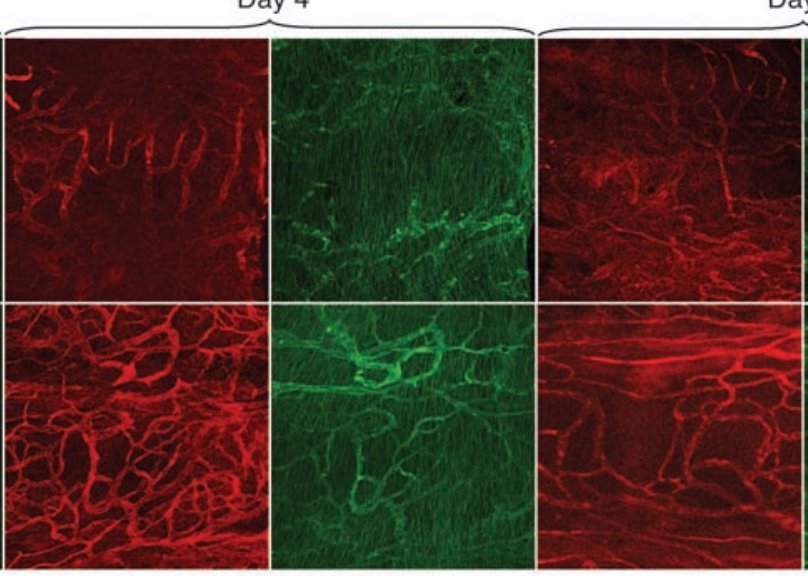

Day 6

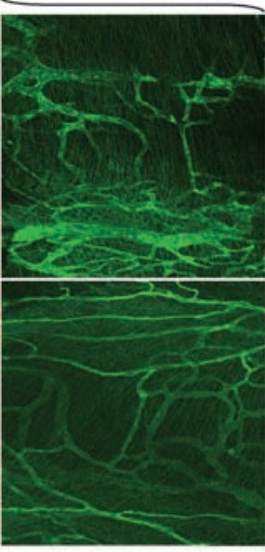

Figure 3

Both allogeneic and syngeneic grafts reperfuse by day 6 via connections of recipient vessels to the preexisting donor vascular network at the anastomosis. (A) At day 2, both allografts and syngrafts have a CD31+ vascular network (red) but no perfusion. Reperfusion of both allografts and syngeneic grafts begins at day 4 and is complete by day 6. (B and C) Mean fluorescent intensity and percent area of FITC ${ }^{+}$vessels. ${ }^{*} P<0.05$ versus both groups at day 2 and day 4 . (D) Schematic of transplanted trachea. Black ovals represent sutures at anastomosis site. Light blue represents the cartilage rings. Red colors the membranous trachea in the transplanted graft, and blue represents that of the recipient ends. $(\mathrm{E}-\mathrm{G})$ Vessel origin experiment was conducted in allografts (BALB/ $\rightarrow \mathrm{FVB}[$ Tie2 / $\beta$-galactosidase]) and syngrafts ( $\mathrm{FVB} \rightarrow \mathrm{FVB}[$ Tie2 / $\beta$-galactosidase]). Prior to tissue harvest, these animals were perfused with red india ink via the aorta to opacify perfused vessels in red. (E) Graft vessels are exclusively red in color (i.e., donor origin). (F) Cutout at anastomosis with suture demonstrating recipient vessels, which connect to donor vessels. Blue-staining cells are noted outside of vessels. (G) Higher magnification at anastomosis. (H) Double stain for $\beta$-galactosidase and CD31 on axial sectioning of day 6 allograft demonstrating that the blue dots are neither part of the CD31 vascular network nor express CD31. (I and J) Serial sections of day 6 allograft independently stained for $\beta$-galactosidase (I) and CD31 (J) to demonstrate that $\beta$-galactosidase-expressing cells do not express CD31 ( $n=3-4$ for all groups). Original magnification, $\times 20(\mathbf{E}, \mathbf{F}, \mathbf{I}$, and $\mathbf{J}) ; \times 40(\mathbf{G}$ and $\mathbf{H})$.

grafts at day 10 and no segments by day 12 (A.N. Babu, unpublished observations). Given that complement activation has been associated with early allograft vascular injury, we queried whether complement-mediated mechanisms could be involved.

C3 deposition is apparent in vascular endothelium of allografts but not syngrafts. Immunofluorescence staining for C3 demonstrated C3 positivity in the subepithelium of allografts, with no staining in syngeneic grafts (Figure 5). Double staining for C3 and CD31 demonstrated colocalization of $\mathrm{C} 3$ with $\mathrm{CD} 31^{+}$vessels, suggesting complement-mediated endothelial injury as one mechanism for vascular destruction.

VEGFR2 and CXC chemokine receptor 2 antagonism do not prevent early angiogenesis but accelerate perfusion loss. To determine what proangiogenic signaling molecules were important in the early revas- cularization of the tracheal allografts, we used pharmaceuticals to antagonize both VEGFR2 and CXC chemokine receptor 2 (CXCR2) signaling independently. Surprisingly, neither strategy affected the early revascularization, and complete revascularization was evident by day 6 in both treatment groups. However, rather than a perfusion loss at day 10, animals treated with VEGFR2 or CXCR2 antagonists had early loss of perfusion, occurring by day 8 (Figure 6).

Untreated allografts develop progressive tissue hypoxia by day 10. Rapid loss of blood supply prior to epithelial sloughing and fibrosis led us to question whether detectable tissue hypoxia was also occurring. To determine whether the loss of perfusion in allografts had a physiologic effect, epithelial tissue oxygen content was measured in the graft in both syngrafts and allografts. Allografts had progressively decreasing tissue partial pressure of $\mathrm{O}_{2}\left(\mathrm{pO}_{2}\right)$ from day $4(25.7 \pm 1.2$ 
A

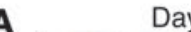

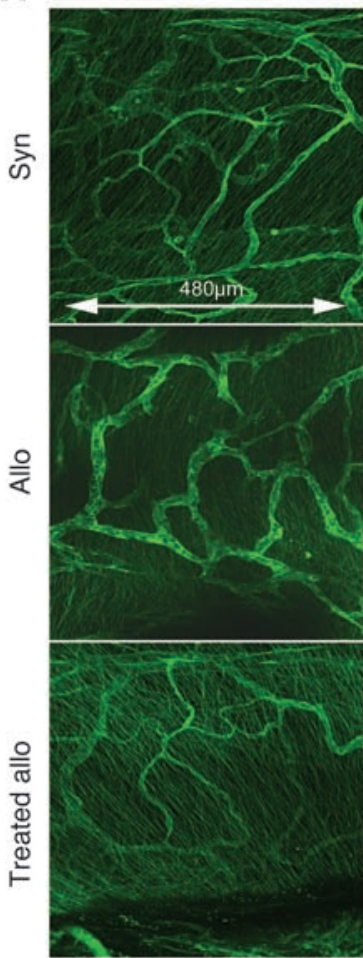

Day 10
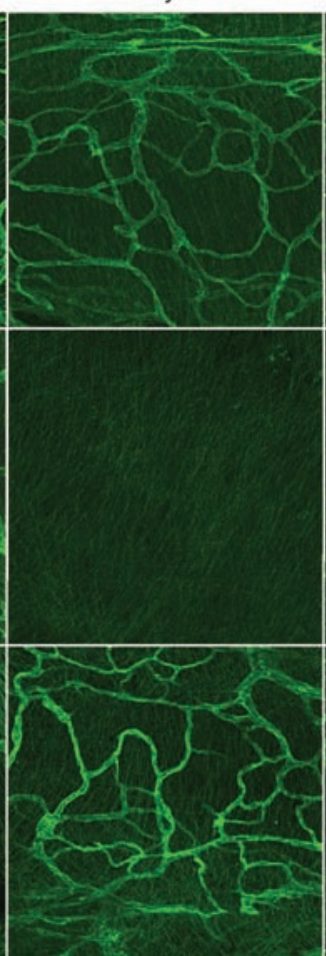

Day 12

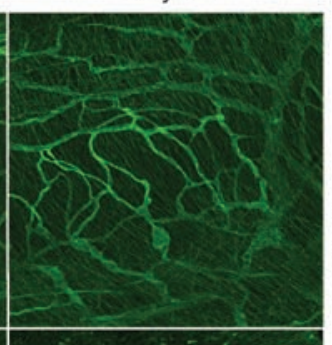

B

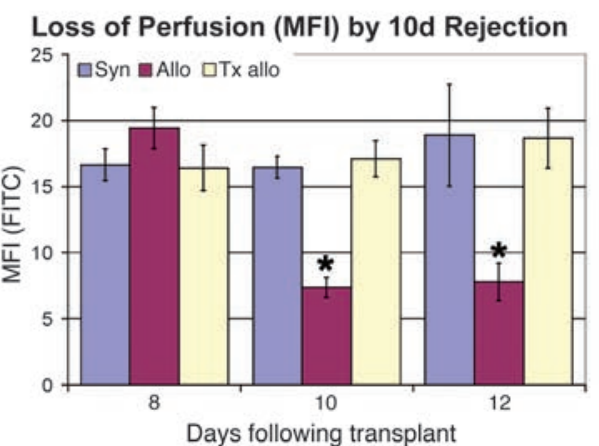

C

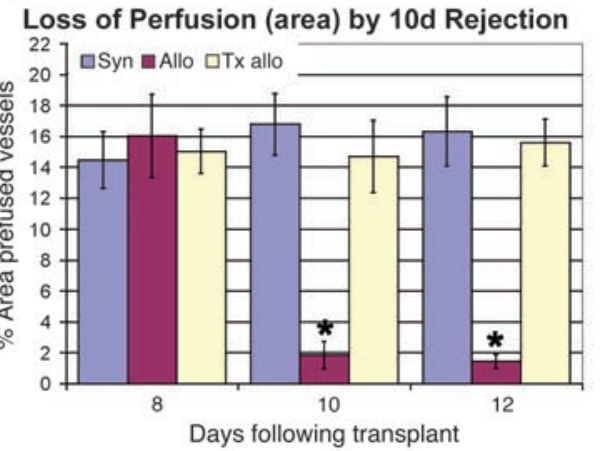

Figure 4

Vascular perfusion is lost by day 10 in untreated allografts and is preserved in syngrafts and immunosuppressed allografts ( $n=3$ per box). (A) Normal vascular perfusion is maintained at day 8 , day 10 , and day 12 in syngrafts. However, there is complete loss of perfusion in allografts at day 10 and day 12. Perfusion of the vessels is completely preserved by immunosuppression at both day 10 and day 12 . Treated allo, allograft treated with immunosuppression (anti-LFA-1 + anti-CD40L). (B) FITC-mean fluorescent intensity was measured as an index of blood flow, confirming the loss of blood flow at day 10 and day 12 in untreated allografts. MFI, mean fluorescent intensity (C) Using a mask based on FITC threshold to mark blood vessels, the percentage area of perfused vessels was assessed to confirm the histologic data. ${ }^{*} P<0.05$ versus all other groups.

$\mathrm{mmHg})$ to day $6(17.2 \pm 0.5 \mathrm{mmHg})$ to day $10(13.3 \pm 1.8 \mathrm{mmHg})$, whereas syngrafts maintained tissue oxygen contents in the range of 32.7 to $36.8 \mathrm{mmHg}$ (Figure $7 \mathrm{~A}$ ).

Only allografts with normal perfusion and endothelium can be rescued by immunosuppression. To determine whether the previously demonstrated perfusion defects had any relevance to the important end point of successful treatment of acute rejection, we employed a delayed immunotherapy model. Allografts $(\mathrm{BALB} / \mathrm{c} \rightarrow \mathrm{C} 57 \mathrm{BL} / 6 \mathrm{~J}$ $[\mathrm{BALB} / \mathrm{c} \rightarrow \mathrm{B} 6])$ underwent rejection for varying durations $(6-14$ days); the graft was then removed and retransplanted into naive B6 animals receiving potent immunosuppression with anti-LFA-1 plus anti-CD40L for 28 days, at which time they were sacrificed. We measured the level of airway remodeling using a previously validated (9) ratio of subepithelial height to epithelial height (Figure 7B). Airways undergoing 6 days of alloimmune injury could be rescued to normal airway architecture. However, tracheae undergoing 10 days or more of alloimmune injury progressed to fibrotic airway remodeling following delayed immunotherapy. On day 6, allografts had massive inflammatory cell infiltrate but normalappearing vascular endothelium according to EM, but appearance was normal after retransplant accompanied by immunosuppression. Day 10 allografts were similarly inflamed; however, the endothelium was markedly abnormal, with thinning of the endothelial barrier, pyknotic nuclei, and red blood cell extravasation visible by EM. Airways with injured endothelium progressed to airway remod- eling with subepithelial fibrosis and epithelial flattening regardless of attempted immunotherapy rescue. To determine changes at the transcriptome level during the period of vascular destruction, day 6 and day 10 allografts were evaluated by gene microarray (Table 1 ). Expression of several genes known to be induced by hypoxia and/or ischemia were increased at day 10 versus day 6 .

An additional transection of vessels during retransplantation leads to ineffective revascularization and subsequent fibrosis. To further demonstrate that the vascular supply was critical to the maintenance of normal airway architecture, the retransplantation studies were again performed following 6 days of alloimmune injury with an additional surgical transection of vessels during retransplantation as illustrated in Figure 8. As described in Figure 7, the BALB/c $\rightarrow$ B6 allograft was cut at its anastomosis, and the $\mathrm{BALB} / \mathrm{c}$ trachea was transplanted to a naive $\mathrm{B} 6$ recipient receiving combined anti-LFA-1/ anti-CD40L therapy. At 10 days following retransplant, normal perfusion to the graft was demonstrated using this technique, and as shown in Figure 7, after 28 days histology exhibited a restoration of normal airway architecture. However, by adding a second transection point on both sides of the allograft in the first recipient, as demonstrated in Figure 8D, perfusion at 10 day was lost in the second immunosuppressed host, and by 28 days the graft had progressed to epithelial flattening and subepithelial fibrosis.

Recipient-derived endothelium contributes to partial revascularization of allograft airway by day 28. Considering the complete loss of perfusion 
A

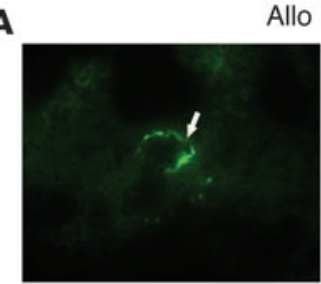

B
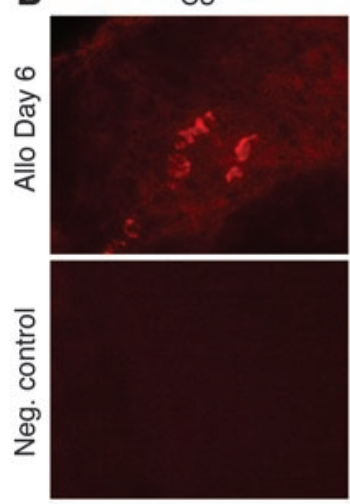

Allo Day 6

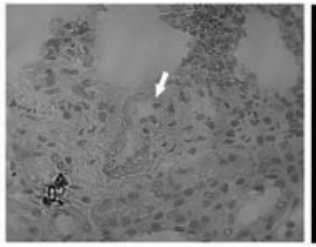

CD31
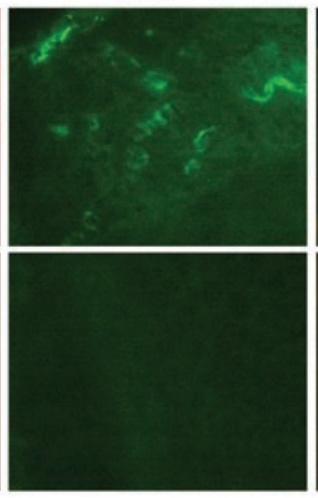

Syn Day 6

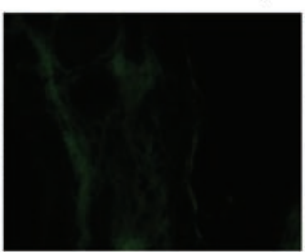

Overlay
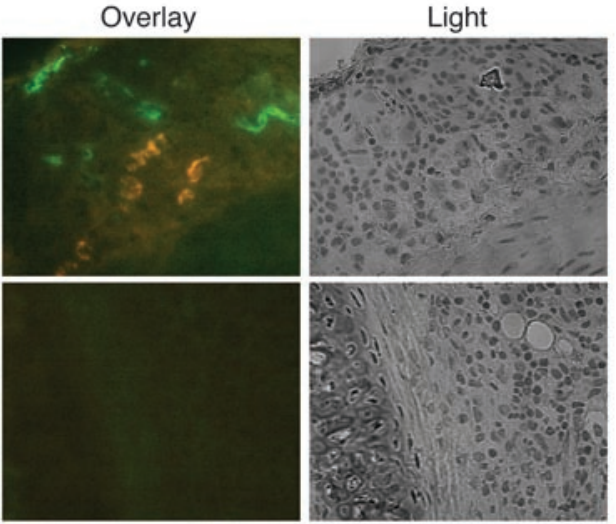

\section{Figure 5}

C3 deposition on endothelium of rejecting allografts. (A) Immunofluorescent (FITC; green) staining for C3 demonstrates positivity in day 6 allografts and none in day 6 syngeneic grafts. (B) Double staining for C3 (Cy3; red) and CD31 (FITC; green) in day 6 allografts demonstrates colocalization (appearing orange) of C3 staining to the vascular endothelium. Original magnification, $\times 40$.

at 10 days, we assessed the 28 day time point in $\mathrm{BALB} / \mathrm{c} \rightarrow \mathrm{C} 57 \mathrm{BL} / 6$ allografts for neovascularization. We did find selective angiogenesis in the central membranous portion of the trachea as well as the intercartilaginous membranous areas in all 4 animals examined (Figure 9). Once again using the FVB (Tie2/ $\beta$-galactosidase) animals as allogeneic recipients, these vessels were noted to partially revascularize the mem- branous trachea and were partially composed of recipient-derived endothelium by 28 days as demonstrated by the black arrows. Interestingly, in 5 of 8 specimens at 28 days, there was columnar epithelium overlying only the membranous portion of the trachea following 28 days of rejection, again suggesting a relationship between regional blood flow and normally differentiated columnar epithelium.

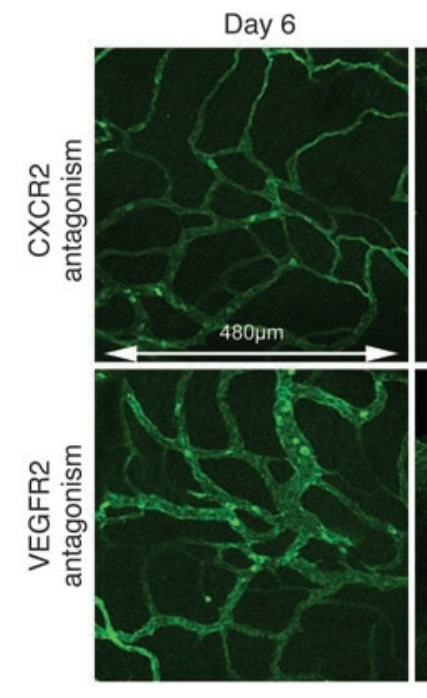

Day 8

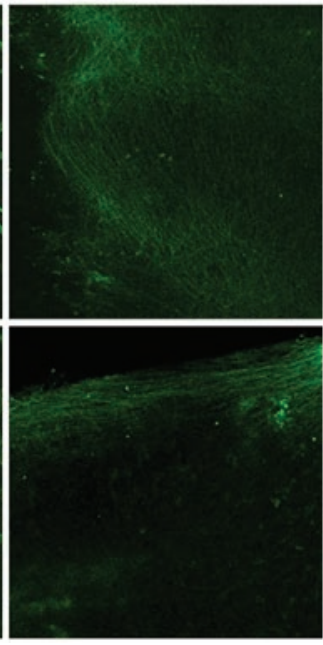

Day 10

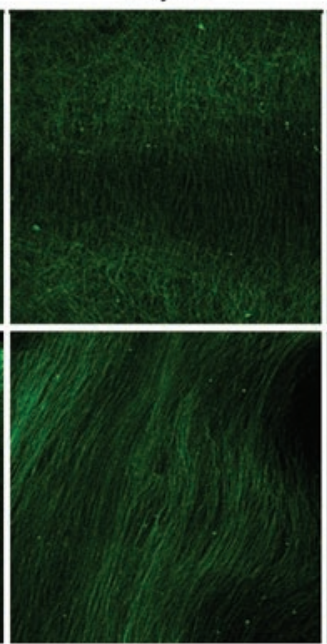

Day 12

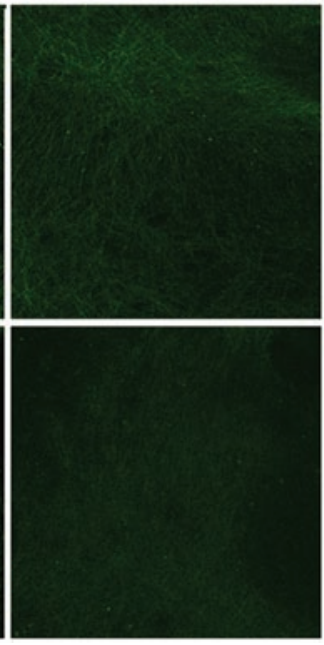

Figure 6

VEGFR2 and CXCR2 antagonism lead to early loss of perfusion. Animals administered a CXCR2 antagonist (antileukinate; $n=3 /$ time point) or a VEGFR2 antagonist (SU5416; $n=3$ /time point) were sacrificed at days $6,8,10$, and 12, and tracheal perfusion was studied by FITC-conjugated lectin perfusion staining. Interestingly, these therapies had no effect on the revascularization occurring by 6 day. However, the loss of perfusion occurred by day 8 rather than by day 10 as in untreated allografts. 
A

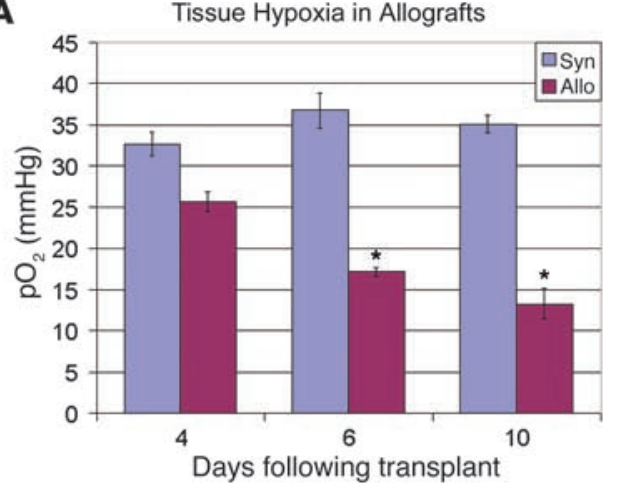

B

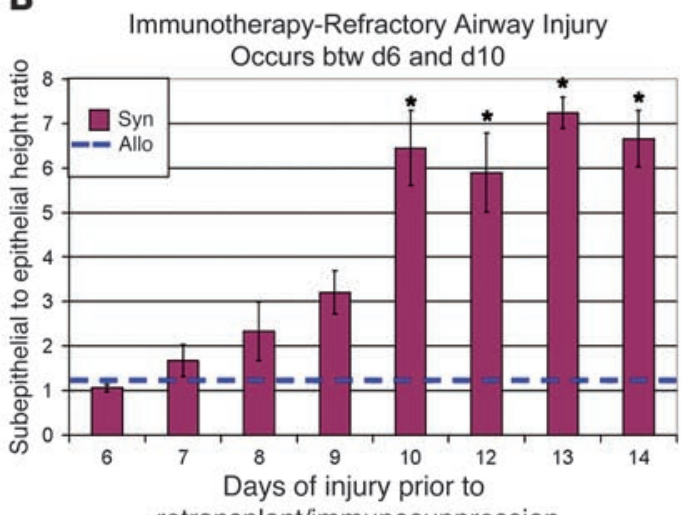

retransplant/immunosuppression
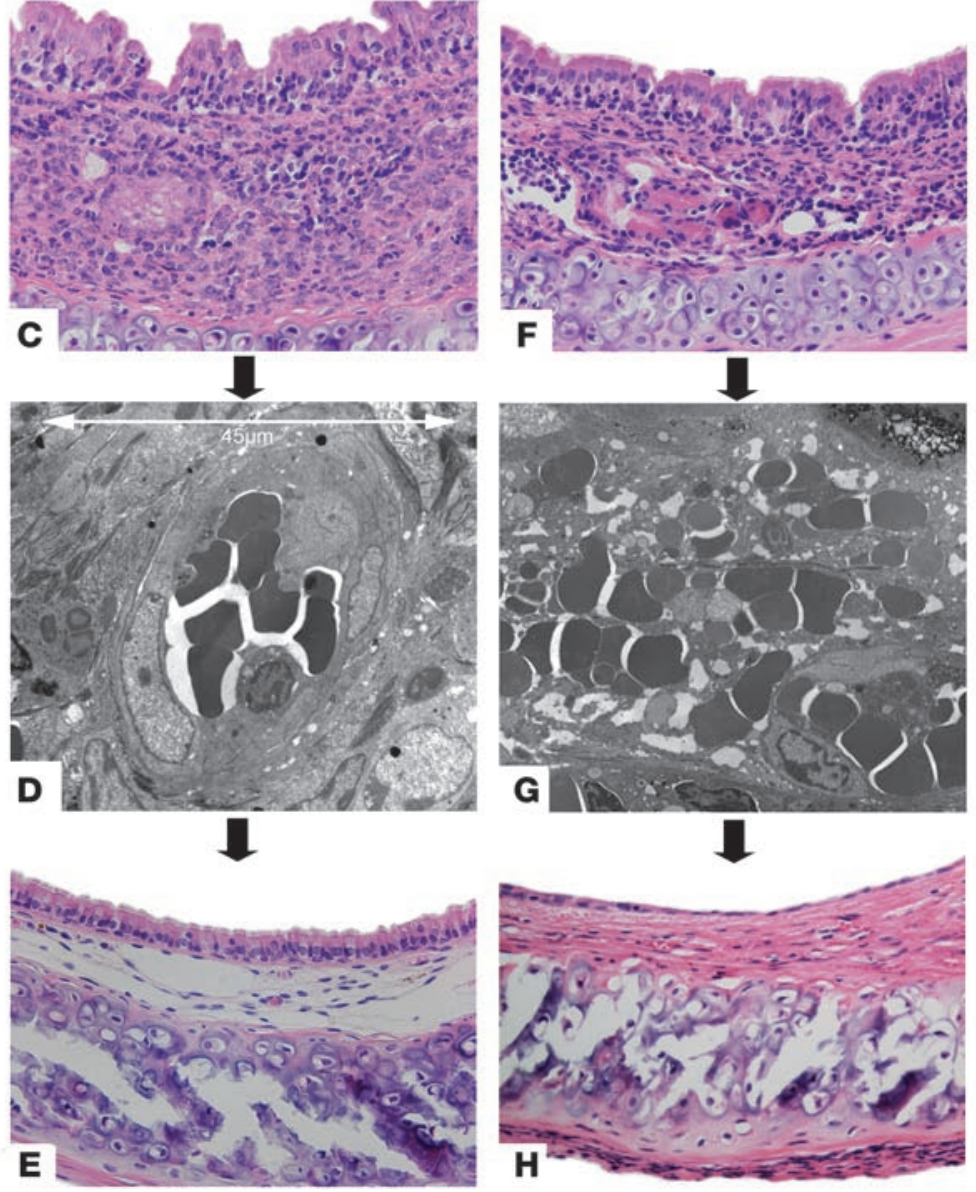

\section{Figure 7}

Injured and nonperfused endothelium is a marker for injury severity that cannot be rescued by immunosuppression. (A) Tissue oximetry was performed using a microprobe to detect tracheal epithelial $\mathrm{pO}_{2}$. Syngrafts maintain consistent tissue oxygen content, whereas allografts develop relative tissue hypoxia by 10 day. (B) Allografts $(B A L B / c \rightarrow B 6)$ were allowed to undergo varying durations of rejection, at which point they were retransplanted to naive immunosuppressed B6 animals for an additional 28 days. H\&E-stained axial sections were used to measure the ratio of subepithelial height to epithelial height. This measure has been validated previously as correlating strongly with chronic rejection histologic scoring (9). Syngeneic grafts that were retransplanted maintained a normal ratio of approximately 1 . Rejection durations of 10 day or longer resulted in severe airway remodeling despite rescue therapy ( $n=5 /$ group). ${ }^{\star} P<0.05$ versus all other groups. (C) Axial section of allograft following 6 days rejection. (D) EM demonstrating normal appearance of vascular endothelium in an allograft following 6 days rejection. (E) Axial section of allograft that underwent 6 days of rejection prior to retransplantation to a naive immunosuppressed animal demonstrating rescue with normal histology. (F) Axial section of allograft following 10 days rejection. (G) EM demonstrating abnormal appearance of vascular endothelium with red blood cell extravasation in an allograft following 10 days rejection. (H) Axial section of allograft that underwent 10 days of rejection prior to retransplantation to a naive immunosuppressed animal demonstrating lack of rescue due to flattened epithelium and subepithelial fibrosis. Original magnification, $\times 20(\mathbf{C}, \mathbf{E}, \mathbf{F}$, and $\mathbf{H}) ; \times 1,100(\mathbf{G})$.

\section{Discussion}

Fibrosis-related organ dysfunction following inflammation limits the success of all organ allografts. Data from this study suggest that a functional microvasculature is critical in the maintenance of normal airway architecture and may be a critical mechanistic link between inflammation and therapy-resistant rejection leading to fibrosis. Loss of $\mathrm{CD} 31^{+}$endothelium after 10 days of airway rejection is synchronous with columnar epithelial sloughing and dysplastic columnar epithelial replacement, a finding that has already been correlated to alloimmune airway fibrosis (9). Following complete revascularization of syngrafts and allografts by 6 days via connection of recipient vessels to preexisting donor vessels in the graft, allografts exhibit complement deposition and undergo immunologically mediated vessel destruction. Loss of functional microvasculature is coincident with the onset of tissue hypoxia and the activation of a hypoxia-induced gene program. The overall relevance of these findings is substantiated by the fact that rejected airways, which still have functional microvasculature, can be rescued by immunotherapy; whereas those with damaged vessels invariably progress to airway remodeling with fibrosis. Additionally, preventing normal revascularization of a rescuable rejected allograft by surgical vessel transection led to fibrosis. This is the first study to our knowledge to link the loss of allograft functional microcirculation to the troublesome clinical entity of immunosuppression-resistant allograft fibrosis.

The use of OTT as a model for alloimmune airway rejection is well established $(9,10)$; airway remodeling is manifested by subepi- 
Table 1

Day 10 versus day 6 array data for angiogenesis-, hypoxia-, and ischemia-related genes

\section{Gene title}

Heme oxygenase (decycling) 1

Heparin-binding EGF-like growth factor

Nitric oxide synthase 2, inducible, macrophage

Plasminogen activator, urokinase

Adrenomedullin

Endothelin 1

Vascular endothelial growth factor $\mathrm{A}$

Tumor necrosis factor receptor superfamily, member $12 a$

Thrombospondin 1

FMS-like tyrosine kinase 1 (VEGFR)

Integrin $\beta 4$

Endothelin receptor type $\mathrm{A}$

NADPH oxidase 4

Angiotensin II receptor, type 2

Gene name
Hmox1
Hbegf
Nos2
Plau
Adm
Edn1
VegfA
Tnfrsf12a
Thbs1
Flt1
Itgb4
Ednra
Nox4
Agtr2

Function
A, I
A
A, H, I
H
$A, H$
I
A, I

$P$ value
0.00006
0.0003
0.00003
0.0003
0.0004
0.00001
0.0002
0.00008
0.0001
0.001
0.0002
0.0006
0.0007
0.00001

Fold change

26.2

18.3

17.6

10.5

10.1

9.5

7.9

6.4

4

3.9

3.3

3.3

$-6$

$-15.9$

RNA isolated from airways rejected for 6 days and 10 days was evaluated by microarray ( $n=4$ per group) for genes related to angiogenesis and those affected by hypoxia and ischemia. Fold changes are calculated as day 10-signal intensity versus day 6 signal intensity. A, angiogenesis; $\mathrm{H}$, hypoxia; I, ischemia.

thelial fibrosis as a result of chronic injury, as occurs in the large airways following human lung transplantation (11). The OTT model is not an obstructive airway disease model but rather reproduces the fibrosis that follows lymphocytic bronchitis. Therefore, the OTT model allows only inferences about the fibrotic process that takes place in terminal bronchioles. The great advantage of this system, however, is that the microvasculature is densely arrayed in a single tissue plane, allowing relatively easy quantitation and description. This is much less readily studied in lung parenchyma or terminal bronchioles, where the anatomy is not so concentrated in a defined area.

The findings of the current model system appear consonant with the observation of small vessel loss prior to the development of BOS and small vessel return (in a limited fashion) in established BOS as noted in lung transplant autopsy studies by Luckraz and colleagues $(4,5)$. These findings may indicate that when inflammation is coupled to relative tissue hypoxemia, immunotherapyrefractory fibroproliferation is more likely to occur than if the tissue is adequately oxygenated. This idea could explain why terminal bronchioles (which are ordinarily partially supplied by the bronchial artery circulation; ref. 12) may be especially vulnerable

\section{Figure 8}

Additional transection of blood vessels during retransplantation affects perfusion and leads to fibrosis. As shown in Figure $7 B, B A L B / C \rightarrow B 6$ allografts having undergone 6 days of rejection can be rescued from fibrosis by retransplantation into a naive B6 immunosuppressed hosts. (A) The standard retransplantation technique involves cutting only the graft from the original recipient at the original anastomosis site and retransplanting it. (B) Ten days following retransplant into naive immunosuppressed host, there is normal perfusion throughout the graft. (C) By 28 days, histology is normal with no fibrosis and well-differentiated epithelium. (D) To determine whether manipulating the blood flow to a rescuable airway could affect end outcome, we divided the day 6 allograft through the recipient ends, creating an additional transection of vessels. (E) At 10 day following retransplant, there was no perfusion, in stark contrast to $\mathbf{B}$. (F) By day 28, this airway had progressed to epithelial flattening and subepithelial fibrosis. Original magnification, $\times 20$ (C, E, and F). to fibrosis in a lung transplant (which has no bronchial artery circulation) compared with other airway inflammatory disorders where the bronchial artery circulation is preserved. It is important to note that with the microvascular changes observed in the Luckraz study (5), even as the vessel count drops and eventually rises, the increased vascularity only involves the relatively low $\mathrm{O}_{2}$ tension of the pulmonary artery circulation.

A recent study by Belperio and colleagues (13) also emphasized the importance of the microvasculature in lung transplantation-
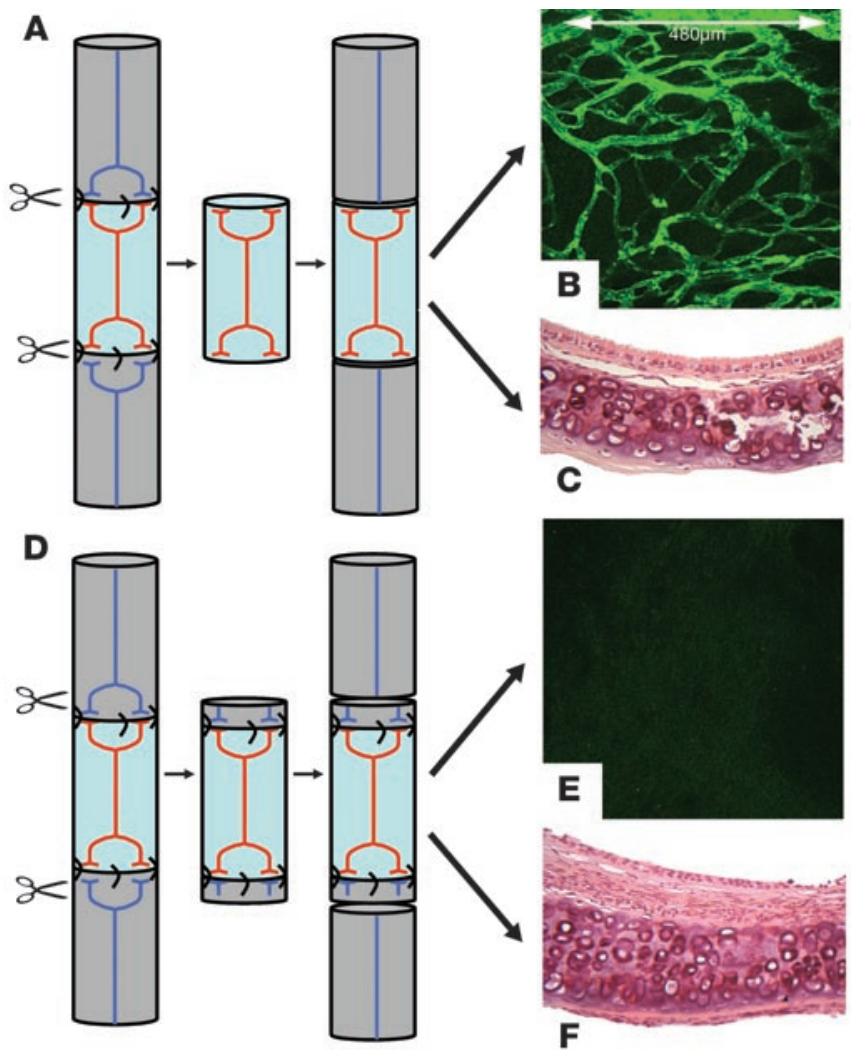


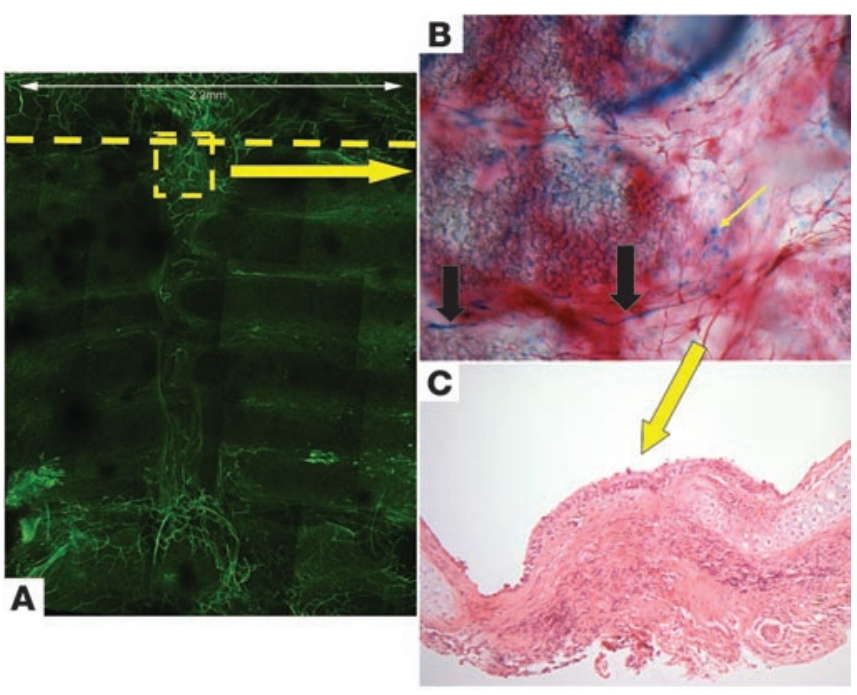

related fibrosis, and is seemingly at odds with our findings as it underscores the potentially deleterious effects of angiogenesis on the development of fibrosis. However, this apparent discrepancy may largely reflect differences in the time points evaluated in each study; microvascular dropout may not have been observed in the former study because it occurred during a noninvestigated time period. The current study demonstrates that angiogenesis occurs early following transplantation, resulting in a hookup of donor and recipient vessels. In keeping with the Luckraz study of clinical lung transplant lungs (5), there is a drop-off in the microvasculature after the connection is lost (analogous to pre-BOS lungs), and then finally there is new angiogenesis after fibrosis is established. Specifically, the current model demonstrates active angiogenesis (on days $4,6,8$, and 28 ) near the time period that the Belperio study demonstrated new angiogenesis (on days 7 and 21). By distinction, the current study also shows a loss of vessels on day 10, which is a time point not examined previously. Indeed, most literature in lung transplantation has reported increased microvasculature in chronic lung transplant rejection $(4,5,14)$. The current study demonstrates that there is a notable period of time when vascular dropout occurs prior to renewed angiogenesis in established fibrosis. While microvasculature destruction has been described in other forms of chronic allograft destruction, it has not been thoroughly examined in lung transplantation, which is likely the graft most burdened by the negative effects of fibrosis. While this concept has been studied to some extent in skin transplantation (15), no published report has examined the therapeutic "point of no return" in relationship to the loss of circulation.

Another important question is in regard to the signaling molecules that cause angiogenesis and vascular hookup at day 6 . VEGF signaling via VEGFR2 is well known to be an important pathway in endothelial cell proliferation and vessel formation (16). VEGF has also been implicated in $\mathrm{T}_{\mathrm{H}} 2$-mediated sensitization, inflammation, and lung parenchymal fibrosis (17). CXC chemokines via their receptor CXCR2 are also known to contribute to angiogenesis in many situations, while CXCR2 antagonism can be protective against experimental fibrosis (13). To determine which pathways were functional in this model, we used drugs to block both pathways. Surprisingly, we did not find any change in the neovascularization occurring by day 6 in treated animals. However, there was

\section{Figure 9}

Neovascularization of membranous trachea by day 28 involves recipient-derived endothelium and supports a well-differentiated epithelium. (A) Neovascularization is evident by perfusion lectin staining in the membranous and intercartilaginous portions of the trachea following 28 days of rejection $(n=4)$. The dashed line indicates the anastomosis site separating the recipient and donor. (B) Use of FVB (Tie2//-galactosidase) recipients $(n=3)$ demonstrated that by day 28 , new vessels were composed partially of recipient-derived endothelium (large black arrows), and $\beta$-galactosidase expressing cells (small yellow arrow) appeared to still be present in the allograft in the proximity of vessels. (C) Five of 8 specimens studied demonstrate columnar epithelium only overlying the membranous portion of the trachea following 28 days of rejection, suggesting that the blood supply in this region may be required to support this epithelial phenotype. Original magnification, $\times 20(B$ and $\mathbf{C})$.

an early loss of perfusion at 8 days in rejecting allografts, suggesting that the neovessels have both VEGFR2 and CXCR2 dependency for their patency. It is possible that these pathways are important for neovascularization as well, but the systemically administered drugs were not perfused to the graft early after transplant. The cumulative findings of this and prior studies are consistent with the seemingly paradoxical notion that increased vascularity to an inflamed airway (influenced in part by accumulating lymphocytes; ref. 18) may be both harmful and protective; angiogenesis may be harmful by facilitating the entrance of leukocytes into the graft but also (at least temporarily) protective in preserving both the nutrient and oxygen supply to a relatively hypoxic area.

A proof-of-principle experiment demonstrating that early vascularity of the allograft is protective is difficult to design because of the overlap between proangiogenic and proimmunogenic factors. Most agents used to address angiogenesis also have immunomodulatory properties. It was therefore necessary to devise a strategy that minimally impacted the immune response of the host or the immunogenicity of the transplant because alteration of either one of these properties would affect the interpretation of results. Due to the difficulty encountered in attempting to induce angiogenesis without immunomodulation, we were forced to explore the converse: that is, we sought to determine whether providing an additional nonimmunomodulatory obstacle to graft revascularization could prevent rescue from fibrosis. To do this, we allowed allografts to undergo 6 days of injury prior to rescue in an immunosuppressed host with or without transections at a secondary site. We reasoned that these new secondary transections provided an additional nonimmunogenic disruption to vessels and could therefore lead to fibroproliferation in the second immunosuppressed recipient in a manner similar to animals that had undergone 10 days of injury. Those grafts that were retransplanted with additional vessel transections were unable to be rescued. While it was assumed that the vascular anastomosis in the first transplant was more tenuous than the native circulation, this was not, in fact, known. Rather, the outcome itself became clear; this second transection resulted in a loss of perfusion to the graft in the second transplant, and this loss of perfusion was again ultimately associated with fibrosis. These data provide additional correlative evidence as to the importance of blood flow in preserving normal graft architecture. 
We defined the time course and mechanism of graft reperfusion in the OTT model using fluorescence perfusion techniques as well as transgenic mice with marked vascular endothelial cells. Reperfusion appeared to occur in an all-or-none pattern consistent with the mechanism of donor vascular network reconnection to recipient vessels. Though this has not been previously studied in orthotopic tracheal grafts, other studies have demonstrated similar mechanics of revascularization as well as time course in avascular grafts such as skin autografts $(19,20)$ and peripheral nerve allografts $(21)$. Interestingly, the transgenic Tie $2 / \beta$-galactosidase recipients had many cells in the allograft expressing $\beta$-galactosidase. However, none of these cells were physically located within blood vessels, nor did they express CD31 antigen. In light of the data demonstrating recipient-derived endothelium contributing to vessels at day 28 , these cells may be immature endothelial progenitor cells that express Tie2 but not CD31 (22). Conversely, these cells may be the result of endothelial-to-mesenchymal transition and could contribute to graft fibrosis (23). Further study is required to completely characterize the phenotype and function of these cells.

The reperfusing chimeric vessels in allografts were destroyed by 10 days following transplantation. Immunologically mediated microvascular destruction in liver allograft bile ducts has been reported to precede the loss of bile ducts, which is characteristic of chronic hepatic rejection (24). Similarly, in renal allografts, severe rejection is characterized by loss of peritubular capillaries, a critical step in graft dysfunction (25). Also, as described above, pre-BOS airways have been demonstrated to have a dearth of microvasculature. Understanding the mechanisms by which allogeneic vessels are injured and not repaired will be critical to designing therapeutic strategies. Vascular endothelial cells present antigen to T lymphocytes, which can induce endothelial cell injury via apoptosis induction $(26,27)$. Additionally, C3 deposition in tracheal vessels at day 6 in the OTT model suggests a role for complement in the vascular injury observed in this model.

Mucosal inflammation in the gut is known to be accompanied by hypoxia and a resulting proangiogenic response (28). Similarly, a proangiogenic mediator profile was also apparent in this model, though immunologic attack of graft vasculature may have prevented an effective response to these mediators. Tissue hypoxia in allografts, as a result of inflammation and vascular destruction, was confirmed by measurement of tissue $\mathrm{pO}_{2}$. Interestingly, allografts at both day 6 and day 10 had relatively low oxygen content. This finding is disparate with the perfusion data showing normal perfusion at day 6 in allografts. However, the presumably high metabolic requirements of the inflammatory cells at day 6 could result in an oxygen supply/demand mismatch, leading to hypoxia even with an intact circulation (28).

Lung transplants are the only solid organ allografts that do not routinely undergo direct systemic arterial reconnection at the time of surgery. One implication of this study is that the absence of the systemic arterial circulation predisposes lung transplant recipients to airway fibrosis. While there is no conclusive evidence currently demonstrating that the lack of a bronchial artery circulation is meaningfully deleterious in lung transplantation, the preponderance of literature indicates that revascularization would likely be a helpful adjunctive maneuver. Preclinical and preliminary clinical studies demonstrate that bronchial artery revascularization improves tissue perfusion with more highly oxygenated blood $(8,29)$; is durable $(30)$, associated with less epithelial metaplasia (31), and protective of pulmonary endothelium and type II pneu- mocytes (32); and may postpone the development of BOS while improving patient survival (33). How hypoxia and ischemia may incite fibrosis is not established. In vitro studies have demonstrated profibrotic phenotypic change of fibroblasts in response to hypoxia (34-36). Epithelial and endothelial cells can undergo mesenchymal transition under ischemia to become another source of activated fibroblasts (37). In summary, although conclusive evidence is lacking, the preponderance of evidence is that revascularization could have a beneficial effect on the success of the transplant. The current study may therefore provide an additional rationale for reconsidering the routine application of this revascularization procedure at the time of lung transplantation.

\section{Methods}

Tracheal transplantation. All animal studies were approved by the University of Colorado Health Sciences Center Institutional Animal Care and Utilization Committee. B6 (H-2 $\left.{ }^{b}\right)$ mice were transplanted with tracheae from B6 and MHC-mismatched BALB/c (H-2 ${ }^{\mathrm{d}}$ ) mice (Jackson Laboratory). Sevenring tracheal segments were removed from $\mathrm{CO}_{2}$-euthanized donor mice that were matched for recipient age and male sex. Recipient mice were anesthetized with ketamine $50 \mathrm{mg} / \mathrm{kg}$ ketamine and $10 \mathrm{mg} / \mathrm{kg}$ xylazine, and a short incision was made in the midline neck region. Division of the strap muscles allowed visualization of the entire laryngotracheal complex. After the recipient's trachea was transected, the donor trachea was sewn in with 10-0 nylon sutures, and the overlying skin was closed with 5-0 silk.

Immunosuppression and drug therapy. The immunotherapy protocol was identical to a previously published study using combined anti-LFA-1/ anti-CD40L therapy (38). Animals received the following protocol: antiLFA-1 (KBA), $200 \mu \mathrm{g}$ i.p. on days $0,1,7$, and 14 (day 0 being the day of transplantation), and anti-CD40L (MR-1), $250 \mu \mathrm{g}$ i.p. on day -1 and twice per week thereafter. VEGFR2 antagonist SU5416 from SUGEN was dissolved in CMC (carboxymethylcellulose) and administered at $20 \mathrm{mg} / \mathrm{kg}$ subcutaneously every other day for the study period. A hexapeptide CXCR2 antagonist known as antileukinate was dissolved in Hanks buffered saline solution to a concentration of $8 \mathrm{mg} / \mathrm{ml}$ and administered using an Alzet microosmotic pump implanted subcutaneously on the day of transplant at a rate of $0.25 \mu \mathrm{l}$ per hour, delivering $2 \mu \mathrm{g}$ of drug per hour (39).

Histology. For axial sections, tracheae were sagittally divided, with onehalf being formalin fixed (H\&E; trichrome) and one-half frozen for immunohistochemistry. Paraffin-embedded tracheal segments were initially fixed in cold $10 \%$ neutral-buffered formalin solution, and $5-\mu \mathrm{m}$ sections were cut and stained for H\&E and Masson's trichrome. For immunohistochemistry studies, $5-\mu \mathrm{m}$ frozen sections were used. Endothelial cells were identified by CD31 immunoreactivity using rat anti-mouse CD31 (BD Biosciences). For class I MHC staining, mouse anti-mouse $\mathrm{H}-2 \mathrm{~K}^{\mathrm{d}}$ (BD Biosciences) was used. An Olympus BX51 microscope with Image-Pro Plus Image Analysis Software (Media Cybernetics) and camera were used for histological and morphometric analysis. Double staining for $\beta$-galactosidase and CD31 involved the standard $\beta$-galactosidase as used below for whole mounts followed by the CD31 immunostaining technique followed above. $\mathrm{C} 3$ staining was conducted on frozen sections using either FITC-conjugated goat anti-mouse $\mathrm{C} 3$ antibody or goat anti-mouse $\mathrm{C} 3$ primary antibody (MP Biomedicals) and a Cy3 rabbit anti-goat secondary antibody (Jackson ImmunoResearch Laboratories Inc.).

Whole-mount tissue fixation, harvest, and staining. Whole-mount tracheae were evaluated for vascular perfusion and CD31 immunoreactivity by fluorescence staining techniques as described previously by Baffert et al. (40). Detailed methods were provided by Donald McDonald (UCSF, San Francisco, California, USA). Under anesthesia, $100 \mu \mathrm{l}$ of $1 \mathrm{mg} / \mathrm{ml}$ FITC-conjugated Lycopersicon esculentum tomato lectin (Vector Laboratories) was injected into the inferior vena cava over 1 minute. After 3 minutes of circulation, 
sternotomy was performed followed by right atriotomy and cannulation of the aorta via left ventricle with an 18 gauge angiocatheter. PBS with $1 \%$ paraformaldehyde was perfused via the aorta for 2 minutes at $120 \mathrm{mmHg}$. Orthotopic tracheal graft along with adjoining recipient trachea was dissected free of surrounding tissues. The trachea was pinned and incubated in $1 \%$ paraformaldehyde. Following a PBS wash, the trachea was bathed in 5\% donkey serum (Jackson ImmunoResearch Laboratories Inc.) and in PBS+ (PBS containing $0.1 \%$ Triton X-100 and $0.2 \%$ albumin). Blocking serum was poured off, and PBS+ containing rat anti-PECAM-1 (CD31) (BD Biosciences) and $1 \%$ donkey serum was added and incubated. Tracheae were then washed in PBS+ and in Cy3-conjugated donkey anti-rat secondary antibody (Jackson Immunoresearch) in PBS+ with no serum and incubated. Whole tracheae were placed on glass slides in Vectashield H1000 anti-quench mounting medium (Vector Laboratories) and a coverslip placed.

Confocal microscopy. Images were acquired on a Zeiss LSM510 laser scanning confocal microscope located in the Light Microscopy Core Facility at the University of Colorado. A software configuration was established to simultaneously capture emission wavelengths from the FITC-conjugated lectin and the Cy3-conjugated secondary antibody used to detect CD31 antigen. Images were captured using a Zeiss $20 \times$ Plan-Apochromat objective with a numerical aperture of 0.8 and a working distance of $0.55 \mathrm{~mm}$. The $x-y$ resolution was $0.45 \mu \mathrm{m}$ and the $z$-stacks were acquired at $2-\mu \mathrm{m}$ intervals. The pinholes for the PMT detectors were optimized to 1 airy disk.

$\beta$-Galactosidase vessel origin study. To identify recipient blood vessels, we used FVB/N-Tg (Tie2-lacZ)182Sato/J mice (Jackson Laboratory), which carry reporter gene $\beta$-galactosidase under control of the Tie 2 (a promoter that identifies vascular endothelium). They were transplanted with tracheae from MHC-matched FVB and MHC-mismatched BALB/c donors. Allogeneic and syngeneic grafts were harvested as whole mounts as described above following $1 \%$ PFA in PBS vascular fixation. They were then soaked in $1 \% \mathrm{PFA}$ in PBS at $4^{\circ} \mathrm{C}$ for 60 minutes. $\beta$-Galactosidase expressing vessels were stained blue using X-Gal Staining kit (Millipore; Chemicon) per the manufacturer's protocol. After rinsing in PBS, tracheae were placed on glass slides with aqueous mounting medium Aqua-Mount (Fisher Scientific) and then compressed with coverslips and 5 ounces lead weight and allowed to dry. As above, Olympus BX51 microscope and camera were used to acquire photomicrographs.

Tissue oximetry. Tissue oxygen content was measured by fluorescence quenching technique using OxyLab $\mathrm{pO}_{2}$ monitor (Oxford Optronix Ltd.) fiber optic probe mounted to a micromanipulator. Trachea was exposed on an anesthetized animal, and 23 gauge needle was used to make an opening in the anterior wall of the trachea. The fiber-optic probe was inserted at a $45^{\circ} \mathrm{C}$ angle to contact the epithelium of the opposite side wall of the trachea. The probe was lowered until it placed gentle pressure on the wall and $\mathrm{pO}_{2}$ reading reduced to $5 \mathrm{mmHg}$ or less (signaling compression of tissue). It was then raised at approximately $20-\mu \mathrm{m}$ increments. The stable $\mathrm{pO}_{2}$ reading, prior to a rapid rise to at least $60 \mathrm{mmHg}$ that signaled loss of tissue contact, was used as the tissue oxygen partial pressure.

EM studies. Tracheal allografts were excised and fixed in $1.5 \%$ glutaraldehyde in $0.1 \mathrm{M}$ cacodylate buffer instilled through the trachea at $23 \mathrm{~cm}$ pressure for 24 hours. Samples were washed with $0.1 \mathrm{M}$ cacodylate buffer, postfixed in $1 \% \mathrm{OsO}_{4}$ buffered with $0.1 \mathrm{M}$ cacodylate for 1 hour, washed again with $0.1 \mathrm{M}$ cacodylate buffer, and stained with $3 \%$ uranyl acetate for 30 minutes. Samples were then dehydrated with grade acetone and embedded in plastic. Cut sections were viewed with a Philips CM10 electron microscope and photographed (Advanced Microscopy Techniques).
Microarray study. To determine how the transcriptome of immune-injured airways compared at critical time points, RNA from tracheal allografts that had undergone 6 days and 10 days of rejection was studied by gene array. Total RNA was extracted from tracheae in TRIzOL reagent and purified using RNeasy (QIAGEN). RNA quality was evaluated with a 2100 Bioanalyzer system (Agilent Technologies). Microarray sample labeling was carried out using the Affymetrix One-Cycle Target Labeling kit for reverse transcription and 1 round of in vitro transcription (41). Affymetrix Mouse Genome 4302.0 microarrays were hybridized with $10 \mu \mathrm{g}$ cRNA and processed per the manufacturer's protocol. Hybridization signals and detection calls were generated in BioConductor (42), using the rma and Affy packages. Microarray data were analyzed using BRB ArrayTools v3.4.1.

Delayed immunotherapy experiments. To determine whether the duration of rejection had any effect on the ability to prevent airway remodeling in acutely rejecting airways, $\mathrm{B} 6$ mice underwent tracheal transplant from $\mathrm{BALB} / \mathrm{c}$ donors. Rejecting grafts were excised from the original recipient. Tracheae were retransplanted orthotopically into immunologically naive B6 mice that received immunotherapy with anti-LFA-1/anti-CD40L as described above. These grafts were re-excised 28 days following retransplantation for histologic examination (38).

Morphometric analysis. Assessment for airway remodeling was performed using a ratio of subepithelial height to epithelial height. We have previously validated this measure as having a high degree of correlation with a chronic rejection scoring system (9). Image-Pro Plus Image Analysis Software (Media Cybernetics) was used to make 3 measurements of each variable in each specimen. Vessel number quantification was performed on axial tracheal sections with CD31 immunostain by counting total numbers of independent $\mathrm{CD} 31^{+}$structures per entire tracheal ring ( $n=5$ per group). Tracheal whole mounts perfused with FITC-conjugated tomato lectin were assessed for mean fluorescent intensity using Intelligent Imaging Innovations SlideBook software. The same software was used to created masks based on a fixed FITC threshold to mask perfused blood vessels. The percentage of area in the mask as compared with total area was measured to create an index of vessel area. Three measurements were made from each specimen.

Statistics. Intergroup statistical analysis was performed using 1-way ANOVA with a Tukey post-test for a significance level of $P<0.05$.

\section{Acknowledgments}

We would like to acknowledge Donald McDonald for providing detailed methods regarding whole trachea staining techniques; Jan Henson for electron microscopy; Steve Fadul and the University of Colorado Health Sciences Center Light Microscopy Core for use of the facilities and training; Fernando Diaz Del Valle for RNA extraction; and the Denver Health Medical Center for use of OxyLab device. This study was supported in part by NIH grants HL082662 (to M.R. Nicolls), HL081655 (to E.J. Miller), DK064790 (to J.M. Thurman), and Amgen Junior Faculty Research Support Program (to J.M. Thurman).

Received for publication April 4, 2007, and accepted in revised form September 12, 2007.

Address correspondence to: Mark R. Nicolls, Division of Pulmonary and Critical Care Medicine, Medical Service (111P), 3801 Miranda Avenue, Palo Alto, California 94304, USA. Phone: (650) 493-5000, ext.69289; Fax: (650) 849-1942; E-mail: mnicolls@stanford.edu.
1. Trulock, E.P., et al. 2006. Registry of the International Society for Heart and Lung Transplantation: twenty-third official adult lung and heart-lung transplantation report - 2006. J. Heart Lung Trans- plant. 25:880-892.

2. Yousem, S.A., Burke, C.M., and Billingham, M.E. 1985. Pathologic pulmonary alterations in longterm human heart-lung transplantation. Hum.
Pathol. 16:911-923.

3. Sharples, L.D., McNeil, K., Stewart, S., and Wallwork, J. 2002. Risk factors for bronchiolitis obliterans: a systematic review of recent publications. 
J. Heart Lung Transplant. 21:271-281.

4. Luckraz, H., et al. 2004. Microvascular changes in small airways predispose to obliterative bronchiolitis after lung transplantation. J. Heart Lung Transplant. 23:527-531.

5. Luckraz, H., et al. 2006. Is obliterative bronchiolitis in lung transplantation associated with microvascular damage to small airways? Ann. Thorac. Surg. 82:1212-1218.

6. Yousem, S.A., Dauber, J.H., and Griffith, B.P. 1990. Bronchial cartilage alterations in lung transplantation. Chest. 98:1121-1124.

7. Pettersson, G., et al. 1994. Early experience of double-lung transplantation with bronchial artery revascularization using mammary artery. Eur. J. Cardiothorac. Surg. 8:520-524.

8. Kamler, M., et al. 2004. Bronchial artery revascularization restores peribronchial tissue oxygenation after lung transplantation. J. Heart Lung Transplant. 23:763-766.

9. Murakawa, T., et al. 2005. Simultaneous LFA-1 and CD40 ligand antagonism prevents airway remodeling in orthotopic airway transplantation: implications for the role of respiratory epithelium as a modulator of fibrosis. J. Immunol. 174:3869-3879.

10. Genden, E.M., Boros, P., Liu, J., Bromberg, J.S., and Mayer, L. 2002. Orthotopic tracheal transplantation in the murine model. Transplantation. 73:1420-1425

11. Yousem, S.A. 1993. Lymphocytic bronchitis/bronchiolitis in lung allograft recipients. Am. J. Surg. Pathol. 17:491-496.

12. Barman, S.A., et al. 1988. Pulmonary and systemic blood flow contributions to upper airways in canine lung. Am. J. Physiol. 255:H1130-H1135.

13. Belperio, J.A., et al. 2005. Role of CXCR2/CXCR2 ligands in vascular remodeling during bronchiolitis obliterans syndrome. J. Clin. Invest. 115:1150-1162. doi:10.1172/JCI200524233.

14. Langenbach, S.Y., et al. 2005. Airway vascular changes after lung transplant: potential contribution to the pathophysiology of bronchiolitis obliterans syndrome. J. Heart Lung Transplant. 24:1550-1556.

15. Schechner, J.S., et al. 2003. Engraftment of a vascularized human skin equivalent. FASEB J. 17:2250-2256.

16. Shibuya, M. 2006. Differential roles of vascular endothelial growth factor receptor-1 and receptor2 in angiogenesis. J. Biochem. Mol. Biol. 39:469-478.

17. Lee, C.G., et al. 2004. Vascular endothelial growth factor (VEGF) induces remodeling and enhances TH2-mediated sensitization and inflammation in the lung. Nat. Med. 10:1095-1103.
18. Auerbach, R., and Sidky, Y.A. 1979. Nature of the stimulus leading to lymphocyte-induced angiogenesis. J. Immunol. 123:751-754.

19. O'Ceallaigh, S., Herrick, S.E., Bluff, J.E., McGrouther, D.A., and Ferguson, M.W. 2006. Quantification of total and perfused blood vessels in murine skin autografts using a fluorescent double-labeling technique. Plast. Reconstr. Surg. 117:140-151.

20. Converse, J.M., Smahel, J., Ballantyne, D.L., Jr., and Harper, A.D. 1975. Inosculation of vessels of skin graft and host bed: a fortuitous encounter. $\mathrm{Br}$. J. Plast. Surg. 28:274-282.

21. Best, T.J., Mackinnon, S.E., Midha, R., Hunter, D.A., and Evans, P.J. 1999. Revascularization of peripheral nerve autografts and allografts. Plast. Reconstr. Surg. 104:152-160.

22. Zengin, E., et al. 2006. Vascular wall resident progenitor cells: a source of postnatal vasculogenesis. Development. 133:1543-1551.

23. Arciniegas, E., Frid, M.G., Douglas, I.S., and Stenmark, K.R. 2007. Perspectives on endothelialmesenchymal transition: potential contribution to vascular remodeling in chronic pulmonary hypertension. Am. J. Physiol. Lung Cell Mol. Physiol. 293:L1-L8.

24. Matsumoto, Y., McCaughan, G.W., Painter, D.M., and Bishop, G.A. 1993. Evidence that portal tract microvascular destruction precedes bile duct loss in human liver allograft rejection. Transplantation. 56:69-75.

25. Bishop, G.A., Waugh, J.A., Landers, D.V., Krensky, A.M., and Hall, B.M. 1989. Microvascular destruction in renal transplant rejection. Transplantation. 48:408-414.

26. Murray, A.G., et al. 1998. Dermal microvascular injury in the human peripheral blood lymphocyte reconstituted-severe combined immunodeficient (HuPBL-SCID) mouse/skin allograft model is T cell mediated and inhibited by a combination of cyclosporine and rapamycin. Am. J. Pathol. 153:627-638.

27. Zheng, L., Gibson, T.F., Schechner, J.S., Pober, J.S., and Bothwell, A.L. 2004. Bcl-2 transduction protects human endothelial cell synthetic microvessel grafts from allogeneic $\mathrm{T}$ cells in vivo. J. Immunol. 173:3020-3026.

28. Karhausen, J., Haase, V.H., and Colgan, S.P. 2005. Inflammatory hypoxia: role of hypoxia-inducible factor. Cell Cycle. 4:256-258.

29. Sundset, A., Tadjkarimi, S., Khaghani, A., Kvernebo, K., and Yacoub, M.H. 1997. Human en bloc double-lung transplantation: bronchial artery revascularization improves airway perfusion. Ann. Thorac. Surg. 63:790-795.
30. Norgaard, M.A., Efsen, F., Andersen, C.B., Svendsen, U.G., and Pettersson, G. 1997. Medium-term patency and anatomic changes after direct bronchial artery revascularization in lung and heart-lung transplantation with the internal thoracic artery conduit. J. Thorac. Cardiovasc. Surg. 114:326-331.

31. Norgaard, M.A., Andersen, C.B., and Pettersson, G. 1999. Airway epithelium of transplanted lungs with and without direct bronchial artery revascularization. Eur. J. Cardiothorac. Surg. 15:37-44.

32. Nowak, K., et al. 2002. Bronchial artery revascularization affects graft recovery after lung transplantation. Am. J. Respir. Crit. Care Med. 165:216-220.

33. Norgaard, M.A., Andersen, C.B., and Pettersson, G. 1998. Does bronchial artery revascularization influence results concerning bronchiolitis obliterans syndrome and/or obliterative bronchiolitis after lung transplantation? Eur. J. Cardiothorac. Surg. 14:311-318.

34. Hong, K.H., et al. 2006. Hypoxia induces expression of connective tissue growth factor in scleroderma skin fibroblasts. Clin. Exp. Immunol. 146:362-370.

35. Karakiulakis, G., Papakonstantinou, E., Aletras, A.J., Tamm, M., and Roth, M. 2007. Cell type-specific effect of hypoxia and platelet-derived growth factor-BB on extracellular matrix turnover and its consequences for lung remodeling. J. Biol. Chem. 282:908-915.

36. Norman, J.T., Clark, I.M., and Garcia, P.L. 2000. Hypoxia promotes fibrogenesis in human renal fibroblasts. Kidney Int. 58:2351-2366.

37. Manotham, K., et al. 2004. Transdifferentiation of cultured tubular cells induced by hypoxia. Kidney Int 65:871-880.

38. Nicolls, M.R., Coulombe, M., Beilke, J., Gelhaus, H.C., and Gill, R.G. 2002. CD4-dependent generation of dominant transplantation tolerance induced by simultaneous perturbation of CD154 and LFA-1 pathways. J. Immunol. 169:4831-4839.

39. Maus, U., et al. 2002. The role of CC chemokine receptor 2 in alveolar monocyte and neutrophil immigration in intact mice. Am. J. Respir. Crit. Care Med. 166:268-273.

40. Baffert, F., et al. 2006. Cellular changes in normal blood capillaries undergoing regression after inhibition of VEGF signaling. Am. J. Physiol. Heart Circ. Physiol. 290:H547-H559.

41. Golpon, H.A., et al. 2004. Emphysema lung tissue gene expression profiling. Am.J. Respir Cell Mol. Biol. 31:595-600.

42. Gentleman, R.C., et al. 2004. Bioconductor: open software development for computational biology and bioinformatics. Genome Biol. 5:R80. 